ARTICLE

\title{
Tailoring the coercive field in ferroelectric metal- free perovskites by hydrogen bonding
}

Hwa Seob Choi ${ }^{1,7}$, Shunning Li (i] 2,7, In-Hyeok Park (B) ${ }^{3}$, Weng Heng Liew ${ }^{4}$, Ziyu Zhu ${ }^{5}$, Ki Chang Kwon (10 1,5, Lin Wang ${ }^{5}$, In-Hwan Oh (1) ${ }^{6}$, Shisheng Zheng ${ }^{2}$, Chenliang Su (1) ${ }^{1}$, Qing-Hua Xu (iD) ${ }^{5}$, Kui Yao (i) ${ }^{4}$, Feng Pan (10) ${ }^{2 \times} \&$ Kian Ping Loh (1D ${ }^{1,5}$

The miniaturization of ferroelectric devices in non-volatile memories requires the device to maintain stable switching behavior as the thickness scales down to nanometer scale, which requires the coercive field to be sufficiently large. Recently discovered metal-free perovskites exhibit advantages such as structural tunability and solution-processability, but they are disadvantaged by a lower coercive field compared to inorganic perovskites. Herein, we demonstrate that the coercive field $(110 \mathrm{kV} / \mathrm{cm})$ in metal-free ferroelectric perovskite MDABCO- $\mathrm{NH}_{4}-\left(\mathrm{PF}_{6}\right)_{3}\left(\mathrm{MDABCO}=\mathrm{N}\right.$-methyl- $\mathrm{N}^{\prime}$-diazabicyclo[2.2.2] octonium $)$ is one order larger than MDABCO- $\mathrm{NH}_{4}-\mathrm{I}_{3}(12 \mathrm{kV} / \mathrm{cm})$ owing to the stronger intermolecular hydrogen bonding in the former. Using isotope experiments, the ferroelectric-to-paraelectric phase transition temperature and coercive field are verified to be strongly influenced by hydrogen bonds. Our work highlights that the coercive field of organic ferroelectrics can be tailored by tuning the strength of hydrogen bonding.

\footnotetext{
${ }^{1}$ SZU-NUS Collaborative Innovation Center for Optoelectronic Science \& Technology, International Collaborative Laboratory of 2D Materials for Optoelectronics Science and Technology of Ministry of Education, Institute of Microscale Optoelectronics, Shenzhen University, 518060 Shenzhen, P.R. China. ${ }^{2}$ School of Advanced Materials, Peking University Shenzhen Graduate School, 518055 Shenzhen, P.R. China. ${ }^{3}$ Graduate School of Analytical Science and Technology (GRAST), Chungnam National University, Daejeon 34134, Republic of Korea. ${ }^{4}$ Institute of Materials Research and Engineering, A*STAR (Agency for Science, Technology and Research), 2 Fusionopolis Way, 138634 Singapore, Singapore. ${ }^{5}$ Department of Chemistry, National University of Singapore, 3 Science Drive 3, 117543 Singapore, Singapore. ${ }^{6}$ Neutron Science Division, Korea Atomic Energy Research Institute, Daejeon 34057, Republic of Korea. ${ }^{7}$ These authors contributed equally: Hwa Seob Choi, Shunning Li. ${ }^{凶}$ email: panfeng@pkusz.edu.cn; chmlohkp@nus.edu.sg
} 
S ince the discovery of ferroelectricity in 1920 in Rochelle salt, enormous research interests have been dedicated to the study of ferroelectrics ${ }^{1}$. Prior to the renaissance of organic ferroelectrics in the past decade, research interests have always been centred on inorganic materials. In contrast to typical inorganic ferroelectric perovskites such as $\mathrm{BaTiO}_{3}$ (BTO) and $\mathrm{Pb}(\mathrm{Zr}, \mathrm{Ti}) \mathrm{O}_{3}(\mathrm{PZT})^{2,3}$, the organic counterparts embrace a unique set of appealing attributes, including high flexibility, fractureresistance, and solution-processability ${ }^{4,5}$. However, the construction of all-organic perovskites is constrained by the types of organic cations and anions that comply with Goldschmidt tolerance factor, which restricts discoveries to a few examples. Among these, the ferroelectric ones are even rarer ${ }^{6,7}$. For example, the recently discovered isostructural family of piperazinium $^{2+}$ all-organic perovskites are mostly non-ferroelectric ${ }^{8,9}$. Achieving ferroelectricity in all-organic perovskites is challenging because polar molecular building blocks generally prefer to adopt an antiparallel arrangement in order to minimize the electrostatic energy of the system ${ }^{10-13}$. One recent breakthrough is the discovery of ferroelectric MDABCO-NH$H_{4}-\mathrm{I}_{3} \quad(\mathrm{MDABCO}=\mathrm{N}$ methyl- $N^{\prime}$-diazabicyclo[2.2.2] octonium) perovskite ${ }^{14}$, whose spontaneous polarization was reported to be comparable to that of BTO. This organic ferroelectric surpasses many inorganic ferroelectrics in terms of parameters such as electrocaloric strength, Curie-Weiss constant and critical field for phase transition ${ }^{15}$. However, a major drawback of MDABCO-NH$-\mathrm{NH}_{3}$ is its low coercive field $(<12 \mathrm{kV} / \mathrm{cm})$. Given that a coercive field larger than $100 \mathrm{kV} / \mathrm{cm}$ is required for voltage switching in the range of $1-2 \mathrm{~V}$ for a $100 \mathrm{~nm}$ thick film ${ }^{16}$, the coercive field of MDABCO- $\mathrm{NH}_{4}-\mathrm{I}_{3}$ would require a thickness of at least $830 \mathrm{~nm}$ to enable operation at $1 \mathrm{~V}$ switching-voltage. This presents a roadblock for the miniaturization of the ferroelectric device. On the other hand, a coercive field higher than $1000 \mathrm{kV} / \mathrm{cm}$ (in PVDFTrFE or $\mathrm{HfO}_{2}$ ) reduces the thickness of the ferroelectric channel to $10 \mathrm{~nm}$, but the drawback is the device is susceptible to a high leakage current, or even dielectric breakdown ${ }^{17,18}$. It is therefore highly desirable to achieve a coercive field on the scale of $100 \mathrm{kV} /$ $\mathrm{cm}$, which demands new design strategies for all-organic ferroelectrics.

Herein, we demonstrate the modulation of the coercive field in organic ferroelectric perovskites via the reinforcement of hydrogen bonding. An all-organic perovskite crystal MDABCO- $\mathrm{NH}_{4}-$ $\left(\mathrm{PF}_{6}\right)_{3}$ was successfully synthesized, where strong $\mathrm{N}-\mathrm{H} \cdots \mathrm{F}$ hydrogen bonds are formed by the $\mathrm{N}-\mathrm{H}$ groups of $\mathrm{MDABCO}$ with the highly electronegative $\mathrm{F}$ (Fig. 1) in $\mathrm{PF}_{6}$. The coercive field of this all-organic compound reaches $110 \mathrm{kV} / \mathrm{cm}$ and its remnant polarization of $5.7 \mu \mathrm{C} / \mathrm{cm}^{2}$ agrees with our Berry phase calculated value of $6.5 \mu \mathrm{C} / \mathrm{cm}^{2}$. The coercive field can be enhanced further to $138 \mathrm{kV} / \mathrm{cm}$ by deuteration, which corroborates the fact that hydrogen bonding influences the coercive field. Our study highlights that coercive field in metal-free perovskites can be enhanced by regulating the strengths of intermolecular hydrogen bonds, suggesting that organic ferroelectrics have wide chemical tunability.

\section{Results and discussion}

As a typical organic perovskite, $\mathrm{MDABCO}-\mathrm{NH}_{4}-\mathrm{I}_{3}$ is composed of divalent amine (MDABCO) at the cuboctahedral centre (Asite), $\mathrm{NH}_{4}{ }^{+}$cation at the octahedral centre (B site) and $\mathrm{I}^{-}$anions occupying the vertices of the octahedron (X site). As MDABCO exhibits the highest remnant polarization among known diamines, we chose it for the A-site cation ${ }^{14}$. To strengthen the hydrogen bond, we replaced $\mathrm{I}^{-}$with $\mathrm{PF}_{6}{ }^{-}$anions (Fig. 2a), the latter is compatible with $\mathrm{NH}_{4}{ }^{+}$and $\mathrm{MDABCO}^{2+}$, yielding a perovskite structure with a tolerance factor of 0.914 (Table S1).

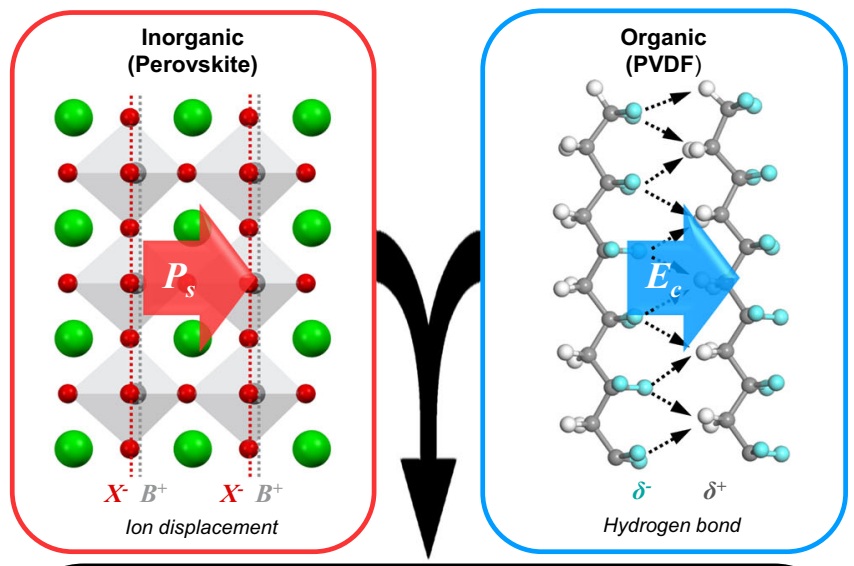

\section{Hydrogen Bonded Organic Perovskite}

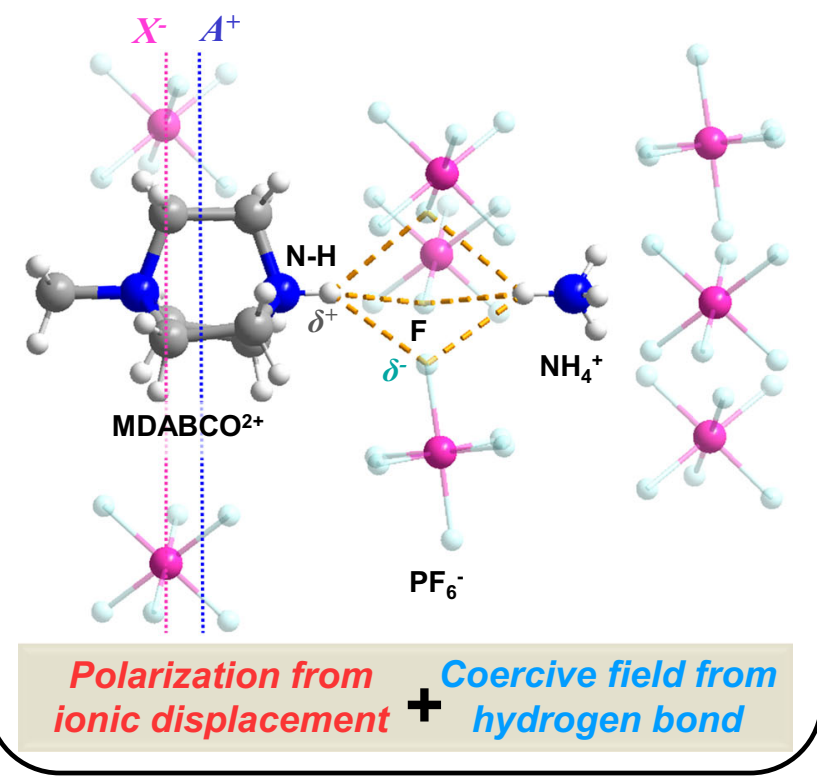

Fig. 1 Schematic of hydrogen-bond-assisted modulation of polarization and coercive field for all-organic ferroelectric perovskites. Red box shows ferroelectric polarization in inorganic perovskite due to ion displacement; Blue box shows ferroelectric polarization due to hydrogen bonds in organic ferroelectric PVDF; Black box shows a combination of both ion

displacement and hydrogen bonds contribute to ferroelectric polarization in organic perovskite.

MDABCO-NH $\mathrm{N}_{4}-\left(\mathrm{PF}_{6}\right)_{3}$ (denoted as $\mathrm{MNP}_{3}$ hereafter) crystal was grown by the slow evaporation of a mixture with a stoichiometric ratio of MDABCO- $\left(\mathrm{PF}_{6}\right)_{2}$ and $\mathrm{NH}_{4} \mathrm{PF}_{6}$, where a single-crystal of $5 \times 4 \times 1 \mathrm{~mm}$ size could be obtained (Fig. 2b) Detailed synthesis procedure is described in Supplementary Information.

The structure of $\mathrm{MNP}_{3}$ was solved by single-crystal X-ray diffraction (SC-XRD), revealing that $\mathrm{MNP}_{3}$ adopts the polar trigonal $R 3$ space group at room temperature (Table S2). The purity of the crystal can be judged from the good agreement between experimental and simulated powder X-ray diffraction (PXRD) patterns (Fig. S1). The overall structure is isostructural to MDABCO-NH ${ }_{4}-\mathrm{I}_{3}\left(\mathrm{MNI}_{3}\right)$, but with a bigger unit cell $(a=b=$ $c=7.844 \AA, \alpha=\beta=\gamma=84.857^{\circ}$ for $\mathrm{MNP}_{3}$ and $a=b=c=$ $7.259 \AA, \alpha=\beta=\gamma=84.767^{\circ}$ for $\mathrm{MNI}_{3}$ ) (Figs. S2, S3). The synthesized crystals are rhombohedral shaped and are $\langle 100\rangle$ textured according to XRD (Figs. S4 and S5). There are multiple hydrogen bonds in $\mathrm{MNP}_{3}$, including $\mathrm{N}-\mathrm{H} \cdots \mathrm{F}$ and $\mathrm{C}-\mathrm{H} \cdots \mathrm{F}$, with bond lengths ranging from 2.2 to $4.0 \AA$ (Figs. S6 and S7). Particularly, 
(a)

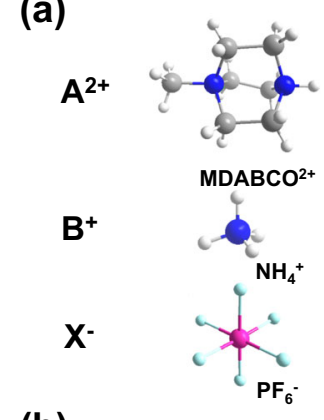

(b)

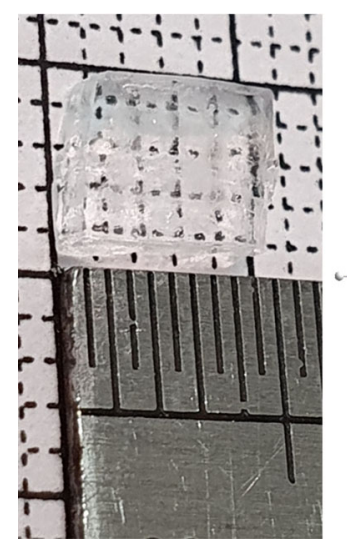

(d)

\begin{tabular}{|c|c|c|c|c|}
\hline & $\begin{array}{c}\text { Unit cell } \\
\text { volume }\left(\AA^{3}\right)\end{array}$ & $d_{111}(\AA ̊)$ & $\delta_{\mathrm{A}}(\mathrm{pm})$ & $\delta_{B}(p m)$ \\
\hline $\mathrm{MNI}_{3}$ & $\mathbf{3 7 7 . 9 8}$ & 13.67 & 65.56 & 0.6366 \\
\hline $\mathbf{M N P}_{3}$ & 477.13 & 14.75 & 73.56 & 0.0312 \\
\hline${\mathrm{D}-\mathrm{MNP}_{3}}_{3}$ & 482.09 & 14.82 & 72.56 & 0.0306 \\
\hline
\end{tabular}

Fig. 2 Crystal structure of MDABCO-NH $-\left(\mathrm{PF}_{6}\right)_{3}$ and the polar displacement direction. a The $\mathrm{A}^{2+}, \mathrm{B}^{+}$and $\mathrm{X}^{-}$components, and their relative positions in $\mathrm{MDABCO}-\mathrm{NH}_{4}-\left(\mathrm{PF}_{6}\right)$ unit cell; $\boldsymbol{b}$ as-grown $5 \times 4 \times 1 \mathrm{~mm}(\mathrm{I} \times \mathrm{d} \times \mathrm{h})$ size single-crystal. c Arrangement of different ions along the [111] polar axis, with arrows indicating the displacement of

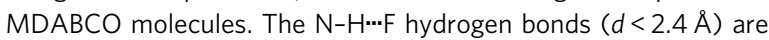
highlighted with dotted orange lines. $\mathbf{d}$ Table of unit cell volume, distance in [111] direction $\left(d_{111}\right)$, displacement of $A^{2+}$ cation $\left(\delta_{A}\right)$, and $B^{+}$cation $\left(\delta_{B}\right)$ for $\mathrm{MNI}_{3}, \mathrm{MNP}_{3}$ and $\mathrm{D}-\mathrm{MNP}_{3}$.

the $\mathrm{N}-\mathrm{H}$ bond of MDABCO is aligned in the [111] polarization direction of $\mathrm{MNP}_{3}$, and form hydrogen bonds with the three nearest fluorine atoms (Fig. 2c). The strong hydrogen bond is revealed from the bond distance, the $\mathrm{N}-\mathrm{H} \cdots \mathrm{F}$ hydrogen bonds (2.4 $\AA$ ) are much shorter than $\mathrm{N}-\mathrm{H} \cdots \mathrm{I}$ in $\mathrm{MNI}_{3}(3.2 \AA)$. The strong $\mathrm{N}-\mathrm{H} \cdots \mathrm{F}$ bond causes MDABCO to be pulled towards [111] direction. As a result, the displacement of MDABCO in the [111] direction is $73.56 \mathrm{pm}$ (4.98\%) for $\mathrm{MNP}_{3}$, which is longer than $\mathrm{MNI}_{3}$ whose displacement is $65.56 \mathrm{pm}$ (4.79\%) (Fig. 2d).

To confirm the effect of hydrogen bond on ferroelectricity, we also synthesized deuterated $\mathrm{MNP}_{3}\left(\mathrm{D}-\mathrm{MNP}_{3}\right)$ in which the proton in $\mathrm{N}-\mathrm{H}$ bond of MDABCO is substituted by deuterium ${ }^{19-21}$. SC-XRD shows that unit cell dimension of D-MNP 3 , with $a=$ $b=c=7.872 \AA$, and $\alpha=\beta=\gamma=84.780^{\circ}$, is slightly larger and shows less octahedral tilt compared to $\mathrm{MNP}_{3}$. This increased unit cell is apparent in the PXRD (Fig. S8). The unit cell dimension $\left(d_{111}\right)$ stretches along the hydrogen-bond direction, i.e. [111] direction, which manifests the Ubbelohde effect ${ }^{22}$. This phenomenon is caused by the longer $\mathrm{N}-\mathrm{D}$ bond than $\mathrm{N}-\mathrm{H}$, so that displacement of MDABCO $\left(\delta_{\mathrm{A}}\right)$ is reduced from $73.56 \mathrm{pm}$ to $72.56 \mathrm{pm}$ by deuteration (Fig. $2 \mathrm{~d}$ ). The longer $\mathrm{N}-\mathrm{D}$ bond is caused by the stronger D-F bonding, which reduces the electron density on $\mathrm{D}$; the distance between the two nitrogen atoms in MDABCO is increased from 2.501 to $2.518 \AA$ by deuteration.

A hallmark of ferroelectricity is the ferroelectric-to-paraelectric phase transition. Phase transition can be detected using differential scanning calorimetry (DSC) measurement, where a sharp endothermic peak can be seen at $311 \mathrm{~K}$ (Fig. $3 \mathrm{a}) . \mathrm{MNP}_{3}$ has a larger cuboctahedral unit cell than $\mathrm{MNI}_{3}$, thus MDABCO in $\mathrm{MNP}_{3}$ can rotate more freely, resulting in a lower phase transition temperature than $\mathrm{MNI}_{3}(448 \mathrm{~K})$. The correlation between free space rotation and phase transition temperature is also demonstrated by the isostructural perovskite MDAB-Rb- $\mathrm{I}_{3}$, where its lower phase transition temperature $(430 \mathrm{~K})$ than $\mathrm{MNI}_{3}$ is correlated to its larger unit cell ${ }^{23}$.

The phase transition temperature of $\mathrm{D}-\mathrm{MNP}_{3}$ is $320 \mathrm{~K}$, which is $19 \mathrm{~K}$ higher than $\mathrm{MNP}_{3}$. The effect of isotope substitution on phase transition temperature is a characteristic feature of hydrogen-bonded ferroelectrics such as KDP family ${ }^{19,24}$, glycine phosfite $^{20}$, and supramolecular complexes ${ }^{4,25,26}$. This implies that all-organic perovskite share characteristics of organic ferroelectrics although the origin of its polarization arises from ionic displacement like inorganic perovskites. The higher phase transition temperature in deuterated perovskite originates from its stronger hydrogen bonds. A stronger hydrogen bond increases the barrier for the rotation of MDABCO in the PE phase similar to $\mathrm{H} / \mathrm{F}$ substituted molecular ferroelectrics ${ }^{27}$. In addition, the much larger enthalpy change of $\mathrm{D}^{-\mathrm{MNP}_{3}}\left(94.5 \mathrm{Jg}^{-1}\right)$ as compared with $\mathrm{MNP}_{3}\left(30.4 \mathrm{~J} \mathrm{~g}^{-1}\right)$ is indicative of the stronger hydrogen bonding in $\mathrm{D}-\mathrm{MNP}_{3}$ as compared to $\mathrm{MNP}_{3}$.

Ferroelectric-paraelectric phase transition was confirmed by a sharp change in second harmonic generation (SHG) intensity across the Curie temperature. The SHG signal originates from the non-centrosymmetric structure of materials, so it vanishes when centrosymmetry is restored in the paraelectric phase. Figure $3 \mathrm{~b}$ shows the temperature-dependent SHG signal of $\mathrm{MNP}_{3}$ and D-MNP 3 collected in the temperature range from 305 to $340 \mathrm{~K}$. The SHG intensity of $\mathrm{MNP}_{3}$ gradually decreases with temperature and vanishes around $315 \mathrm{~K}$ due to the recovery of centrosymmetry in the paraelectric phase. Similarly, the SHG signal of $\mathrm{D}-\mathrm{MNP}_{3}$ vanishes around $323 \mathrm{~K}$ when the crystal transits to the centrosymmetric phase, in line with the DSC results.

$\mathrm{MNP}_{3}$ and $\mathrm{D}-\mathrm{MNP}_{3}$ undergo a phase transition from the ferroelectric $R 3$ space group to the paraelectric cubic $P 432$ space group similar to what has been reported for isostructural perovskite structures ${ }^{14,23}$. The paraelectric unit cell dimensions of $\mathrm{MNP}_{3}$ are $a=b=c=7.9557 \AA$, and $\alpha=\beta=\gamma=90^{\circ}$, and for paraelectric D-MNP $3, a=b=c=7.9154 \AA$, and $\alpha=\beta=\gamma=90^{\circ}$ which confirmed by SC-XRD. To monitor structural changes during the phase transition process, temperature-dependent powder X-ray diffraction (PXRD) for both $\mathrm{MNP}_{3}$ and $\mathrm{D}-\mathrm{MNP}_{3}$ were recorded. At the transition temperature of each sample, the XRD peaks of (100), (111) and (200) at $11.26^{\circ}, 20.14^{\circ}$ and $22.68^{\circ}$ (Fig. S9) shift to lower angles of $11.12^{\circ}, 19.36^{\circ}$ and $22.4^{\circ}$, respectively, indicating that the unit cell dimension has expanded; the vanishing of other peaks indicate that a more symmetric cubic cell is achieved in paraelectric phase. It is observed that the (111) peaks due to ferroelectric phase at $20.14^{\circ}$ and the paraelectric phase at $19.36^{\circ}$ coexist without other intermediate states during phase transition (Fig. $3 \mathrm{c} 313 \mathrm{~K}$ and $3 \mathrm{~d} 323 \mathrm{~K}$ ), this suggests that the phase change is a discontinuous order-disorder type (firstorder $^{28}$.

The fingerprints of ferroelectric-to-paraelectric phase transition can also be observed in the solid-state ${ }^{1} \mathrm{H}$ NMR spectrum (Fig. 3e). The $\mathrm{CH}_{2}$ and $\mathrm{CH}_{3}$ peaks of $\mathrm{MDABCO}$ at 3.9 and 3.2 
(a)

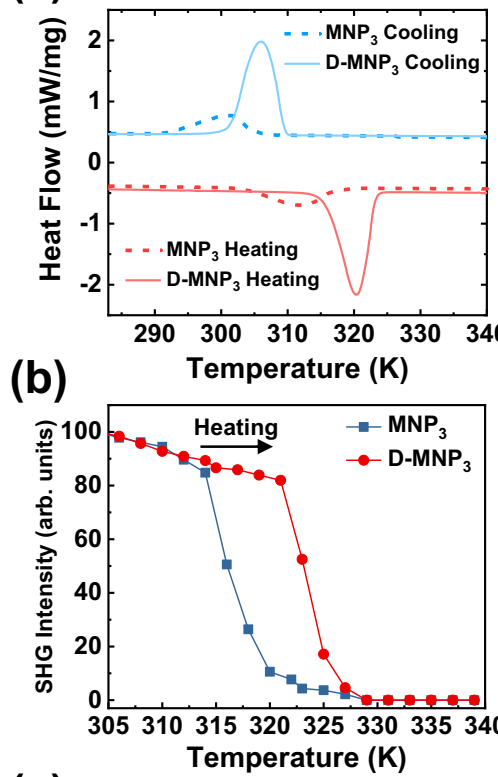

(e)

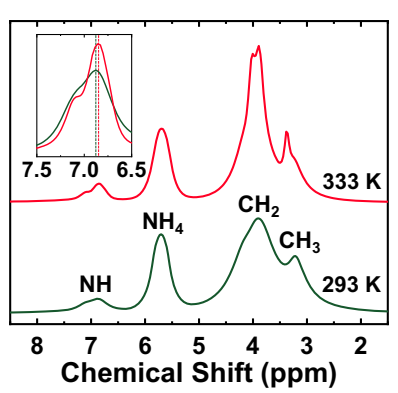

(c)

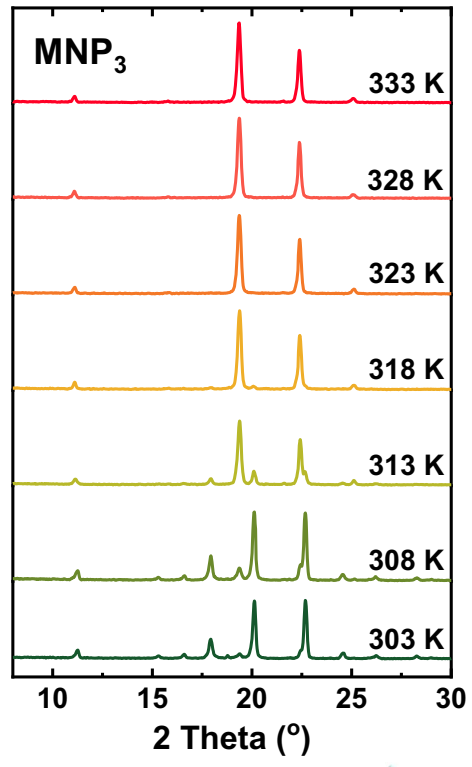

(d)

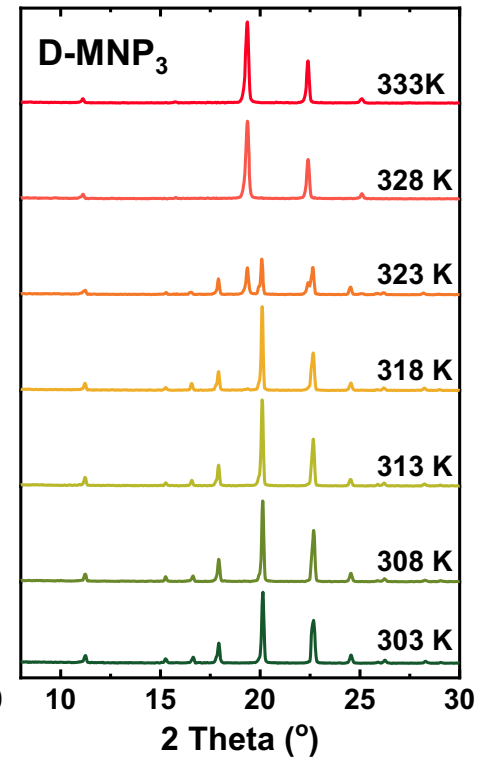

(f)

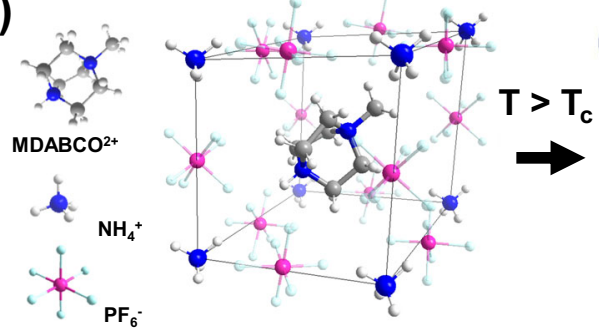

FE Phase

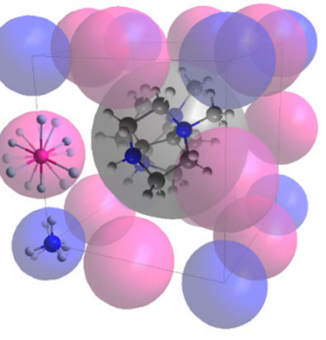

PE Phase

Fig. 3 The ferroelectric-to-paraelectric transition of $\mathbf{M N P}_{\mathbf{3}}$ and $\mathbf{D}-\mathbf{M N P}_{\mathbf{3}}$ crystal. a DSC and $\mathbf{b}$ temperature-dependent SHG intensity change of $\mathrm{MNP} \mathrm{P}_{3}$ and $D-M N P_{3}$ revealing phase transition from ferroelectric (FE) phase to paraelectric ( $P E$ ) phase, and that D-MNP $P_{3}$ has a higher $T_{c}$ than $M N P_{3}$. Temperaturedependent PXRD result of $\mathrm{MNP}_{3}$ (c) and $\mathrm{D}-\mathrm{MNP}_{3}$ (d). e Solid-state ${ }^{1} \mathrm{H}$ NMR spectrum of $\mathrm{MNP}_{3}$ at $293 \mathrm{~K}$ (FE phase) and $333 \mathrm{~K}$ (PE phase). Inset shows peak shift (6.88-6.84) of $\mathrm{N}-\mathrm{H}$ in MDABCO indicating shrinkage of $\mathrm{N}-\mathrm{H}$ bond. $\mathbf{f}$ Schematic illustration of $\mathrm{MNP}_{3}$ structure in ferroelectric (FE) phase and paraelectric (PE) phase. In the PE phase, molecules rotate freely.

ppm become sharper in the paraelectric phase due to the free rotation of the MDABCO molecule. The peak of $\mathrm{N}-\mathrm{H}$ of MDABCO at 6.88 is split into two due to a second-order effect of dipolar coupling to the quadrupolar nitrogen-14 nuclei ${ }^{29}$. This peak shift from 6.88 to $6.84 \mathrm{ppm}$ that accompanies phase transition is indicative of a reduction of the $\mathrm{N}-\mathrm{H}$ bond length because of the weakening of the hydrogen bonds in the paraelectric phase $^{30}$. Furthermore, the $\mathrm{P}-\mathrm{F}$ distance is also shortened in the paraelectric phase at $333 \mathrm{~K}$ as reflected from the solid-state NMR, where the J-coupling constant is reduced (Fig. S10). Therefore, solid-state NMR results validate that $\mathrm{N}-\mathrm{H}$ and $\mathrm{P}-\mathrm{F}$ bonds are elongated in the ferroelectric phase due to strong hydrogen bonds, whereas in the $\mathrm{PE}$ phase, both $\mathrm{MDABCO}$ and $\mathrm{PF}_{6}$ rotate freely and $\mathrm{N}-\mathrm{H}$ and $\mathrm{P}-\mathrm{F}$ bonds are shorter. The orientational disorder of molecules in the paraelelectric phase has been extensively studied previously by Jun Harada and others ${ }^{31-35}$. One characteristic of this rotation disorder is that under polarized light microscopy, the iridescence of the ferroelectric phase due to birefringence vanishes upon changing to the paraelectric phase, which is clearly confirmed for our $\mathrm{MNP}_{3}$ crystal (supplementary information Fig. S11).

Temperature-dependent dielectric permittivity was also used to detect the phase transition of the crystals as a ferroelectric-to paraelectric transition will result in an anomaly in the dielectric permittivity. Both $\mathrm{MNP}_{3}$ and $\mathrm{D}-\mathrm{MNP}_{3}$ show an abrupt increase of dielectric permittivity around their phase transition temperature (Fig. S12 a and b). We also fitted the inverse of dielectric constant with the temperature at $100 \mathrm{kHz}$ (Fig. S12 c and d) by Curie-Weiss law that is, $\varepsilon=C /\left(T-T_{0}\right)$ where $\varepsilon$ is the dielectric constant, $\mathrm{C}$ is the Curie constant, $\mathrm{T}$ is the temperature, and $\mathrm{T}_{0}$ is the Curie temperature. Linear fitting of $1 / \varepsilon$ with the temperature at $100 \mathrm{kHz}$ allows the Curie temperatures of $\mathrm{MNP}_{3}$ and $\mathrm{D}-\mathrm{MNP}_{3}$ to be determined as $307 \mathrm{~K}$ and $316 \mathrm{~K}$, and the Curie constants as $254 \mathrm{~K}$ and $382 \mathrm{~K}$, respectively. The dielectric permittivity around the phase transition temperature is strongly dependent on frequency. A smaller dielectric permittivity at a higher frequency means the ferroelectric-to-paraelectric transition is the orderdisorder type ${ }^{36}$. D- $\mathrm{MNP}_{3}$ shows a much higher dielectric permittivity than $\mathrm{MNP}_{3}$, which may be explained by the higher polarization of the former arising from its stronger $\mathrm{H}$-bonding ${ }^{37}$.

The polarization $(P)$ versus electric field $(E)$ measurement as shown in Fig. 4a exhibits a hysteresis loop characteristic of a ferroelectric crystal. The remnant polarization $P_{\mathrm{r}}$ is $5.7 \mu \mathrm{C} / \mathrm{cm}^{2}$, and the coercive field $E_{\mathrm{c}}$ ranges from 54 to $110 \mathrm{kV} / \mathrm{cm}$ depending on the applied frequency. The coercive field of $\mathrm{MNP}_{3}$ is much higher than isostructural organic perovskite $\mathrm{MNI}_{3}(12 \mathrm{kV} / \mathrm{cm})$, this is in line with the expectation that a stronger hydrogen bond leads to a higher coercive field. Compared with the relatively low coercive field of BTO $(10 \mathrm{kV} / \mathrm{cm})$ and PZT $(76 \mathrm{kV} / \mathrm{cm})^{38}$, and the exceedingly large field of $500 \mathrm{kV} / \mathrm{cm}$ for $\mathrm{PVDF}^{39}, \mathrm{MNP}_{3}$ exhibits 
(a)

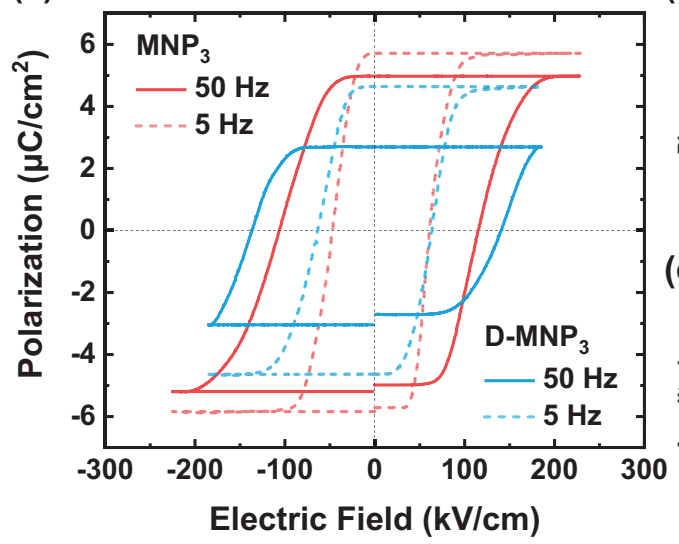

(b)

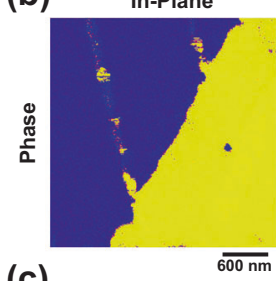

(c)

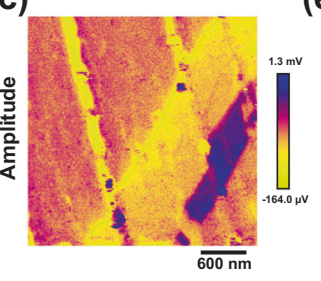

(d)

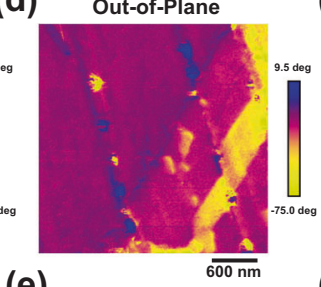

(e)

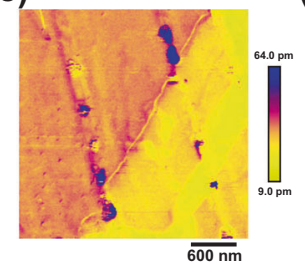

(f)

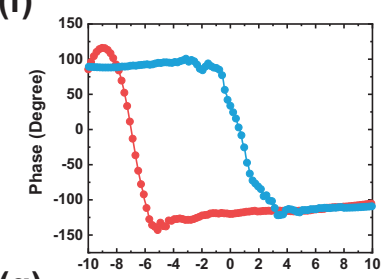

(g)

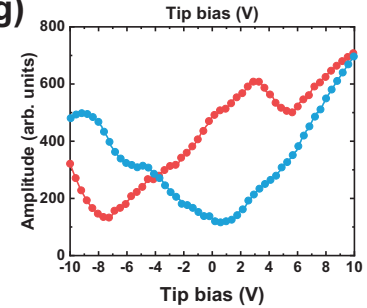

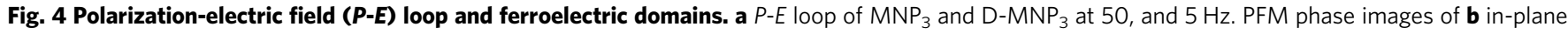

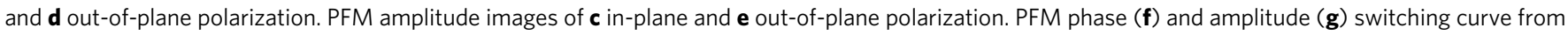
$\mathrm{MNP}_{3}$ thin film.

a mid-range coercive field that translates to a threshold voltage of $1 \mathrm{~V}$ for a $90 \mathrm{~nm}$ thick film, which is compatible with the standard operating condition of thin-film ferroelectric random-access memory (FeRAM) devices. Furthermore, the coercive field of $\mathrm{D}-\mathrm{MNP}_{3}$ is increased to $138 \mathrm{kV} / \mathrm{cm}$ at $50 \mathrm{~Hz}$, this agrees with the fact that $\mathrm{D}-\mathrm{MNP}_{3}$ has stronger hydrogen bonds. These results prove that we can control the coercive field of the ferroelectric materials by tuning the intermolecular hydrogen-bond strength.

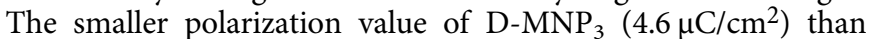
$\mathrm{MNP}_{3}\left(5.7 \mu \mathrm{C} / \mathrm{cm}^{2}\right)$ at $5 \mathrm{~Hz}$ can be attributed to two factors: first,

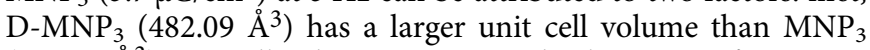
$\left(477.13 \AA^{3}\right)$; secondly, the A-site cation displacement of D-MNP $(72.56 \mathrm{pm})$ is shorter than $\mathrm{MNP}_{3}(73.56 \mathrm{pm})$ due to the Ubbelohde effect. The polarization values at $5 \mathrm{~Hz}$ differ by about $23 \%$, but it increases to $84 \%$ at $50 \mathrm{~Hz}$ where the polarization value of $\mathrm{D}-\mathrm{MNP}_{3}$ is $2.7 \mu \mathrm{C} / \mathrm{cm}^{2}$ and $\mathrm{MNP}_{3}$ is $5.0 \mu \mathrm{C} / \mathrm{cm}^{2}$. Considering that the decline in polarization at high frequency is due to the inability of the switching dipoles to keep up with the oscillating field, this effect is more apparent in $\mathrm{D}-\mathrm{MNP}_{3}$ due to its stronger hydrogen bond. (Fig. S13).

According to the $432 \mathrm{F3}$ transition, $\mathrm{MNP}_{3}$ has four polar axis and eight polarization directions ${ }^{40}$. Since the polar axes are in between the in-plane ( $y$-axis) and out-of-plane ( $z$-axis) directions, ferroelectric domains whose polarization directions are not perpendicular to these can be observed in both in-plane and out-ofplane phase and amplitude images using piezoresponse force microscopy (PFM). In Fig. $4 \mathrm{~b}$, a $180^{\circ}$ in-plane domain change can be seen clearly, which indicates two polarized domains in the $y$ axis direction $(+y$ and $-y)$. In the amplitude scan of the in-plane PFM image (Fig. 4c), there is a region that overlaps with some parts of the out-of-plane phase (Fig. 4d), which is indicative of canted polarizations with vectors in both $-\mathrm{y}$ and $-\mathrm{z}$ directions (Fig. S14 b green area). We could also identify areas (red in Fig. S14) with canted polarizations and vector components in $-y$ and $-\mathrm{z}$ direction, and another two regions with canted polarizations and vector components along $+y$ and $-y$ region according to the in-plane phase image. Therefore, from the PFM images, we can observe four distinct polarization domains (Fig. S14 b and c), similar to the previously reported isostructural $\mathrm{MNI}_{3}{ }^{14}$. Due to the high coercive field of $\mathrm{MNP}_{3}$, it was difficult to perform phase switching on a thick, single-crystal sample using PFM. Instead, ferroelectric switching was performed on a $1 \mu \mathrm{m}$-thick thin film of $\mathrm{MNP}_{3}$ prepared by a spray-coating method (Fig. S15). A clear 180-degree phase reversal (Fig. 4f) and butterfly curve (Fig. 4g) were observed by sweeping the tip voltage from -10 to $10 \mathrm{~V}$, indicating that ferroelectric domains can be electrically switched.

To validate our experimentally measured polarization values and coercive field, we performed density functional theory (DFT) calculations of both $\mathrm{MNI}_{3}$ and $\mathrm{MNP}_{3}$. First of all, spontaneous polarization value was calculated by Berry phase calculation developed by King-Smith and Vanderbilt ${ }^{41,42}$. We constructed a $\sqrt{ } 2 \times \sqrt{2} \times 1$ supercell based on the unit cell of the ferroelectric phase, and built the centrosymmetric reference phase via rotation, displacement and distortion of the components, after which two MDABCO molecules in the simulation cell are aligned antiparallel to each other (Fig. 5a). The calculated polarization of $\mathrm{MNP}_{3}$ is shown in Fig. 5b, where it varies continuously from 0 to $6.5 \mu \mathrm{C} / \mathrm{cm}^{2}$ along the dynamic path, thus the spontaneous polarization agrees well with the experimental value of $5.7 \mu \mathrm{C} /$ $\mathrm{cm}^{2}$. Although the larger displacement of $\mathrm{MDABCO}$ in $\mathrm{MNP}_{3}$ than $\mathrm{MNI}_{3}$ (Fig. 2d) gives a larger dipole moment in the former, the larger unit cell volume of $\mathrm{MNP}_{3}$ than $\mathrm{MNI}_{3}$ offsets the increased polarization as the overall polarization is defined by the sum of dipole moment per unit volume. Furthermore, in $\mathrm{MNI}_{3}$, there is a large polarization contribution from the off-centre displacement of $\mathrm{NH}_{4}{ }^{+}$at $\mathrm{B}$ site $\left(\delta_{\mathrm{B}}=0.6366 \mathrm{pm}\right)$ because of its larger $\mathrm{r}_{\mathrm{B}} / \mathrm{r}_{\mathrm{X}}$ ratio $(0.664)$ compared to the typical octahedron ratio (0.414-0.592) predicted by Pauling's rule. On the other hand, the $\mathrm{NH}_{4}+$ ion in $\mathrm{MNP}_{3}$ is located at the centre of the octahedron $\left(\delta_{\mathrm{B}}=0.0312 \mathrm{pm}\right)$ with a $\mathrm{r}_{\mathrm{B}} / \mathrm{r}_{\mathrm{X}}$ ratio of 0.570 , thus there is no offcentre displacement.

The displacement of $\mathrm{MDABCO}$ in the polarization direction with respect to the $\mathrm{MNP}_{3}$ framework is the primary driver of the ferroelectricity of $\mathrm{MNP}_{3}$. The large electrostatic potential differences between $\mathrm{MDABCO}-\mathrm{NH}$ and $\mathrm{PF}_{6}$ increase the hydrogenbond strength compared to $\mathrm{MNI}_{3}$. Mulliken charge calculations for $\mathrm{MNP}_{3}$ and $\mathrm{MNI}_{3}$ reveal that $\mathrm{PF}_{6}-$ has a more pronounced inductive effect than $\mathrm{I}^{-}$on the $\mathrm{H}$ atom in MDABCO (Fig. 5c), thus the stronger $\mathrm{N}-\mathrm{H} \cdots \mathrm{F}$ hydrogen retards the polarity reversal process of organic ferroelectric perovskites. Therefore, any reinforcement of hydrogen bonding will hinder the ferroelectric switching process, since multiple hydrogen bonds need to be successively broken and established during the rotation of MDABCO, as illustrated in Fig. 5a. This is the root cause of the enhanced coercive field for $\mathrm{MNP}_{3}$.

In conclusion, incorporating stronger hydrogen bonds in MDABCO- $\mathrm{NH}_{4}-\mathrm{I}_{3}$ by the substitution of $\mathrm{PF}_{6}{ }^{-}$for $\mathrm{I}^{-}$increase the coercive field by an order of magnitude from $\sim 12 \mathrm{kV} / \mathrm{cm}$ to 

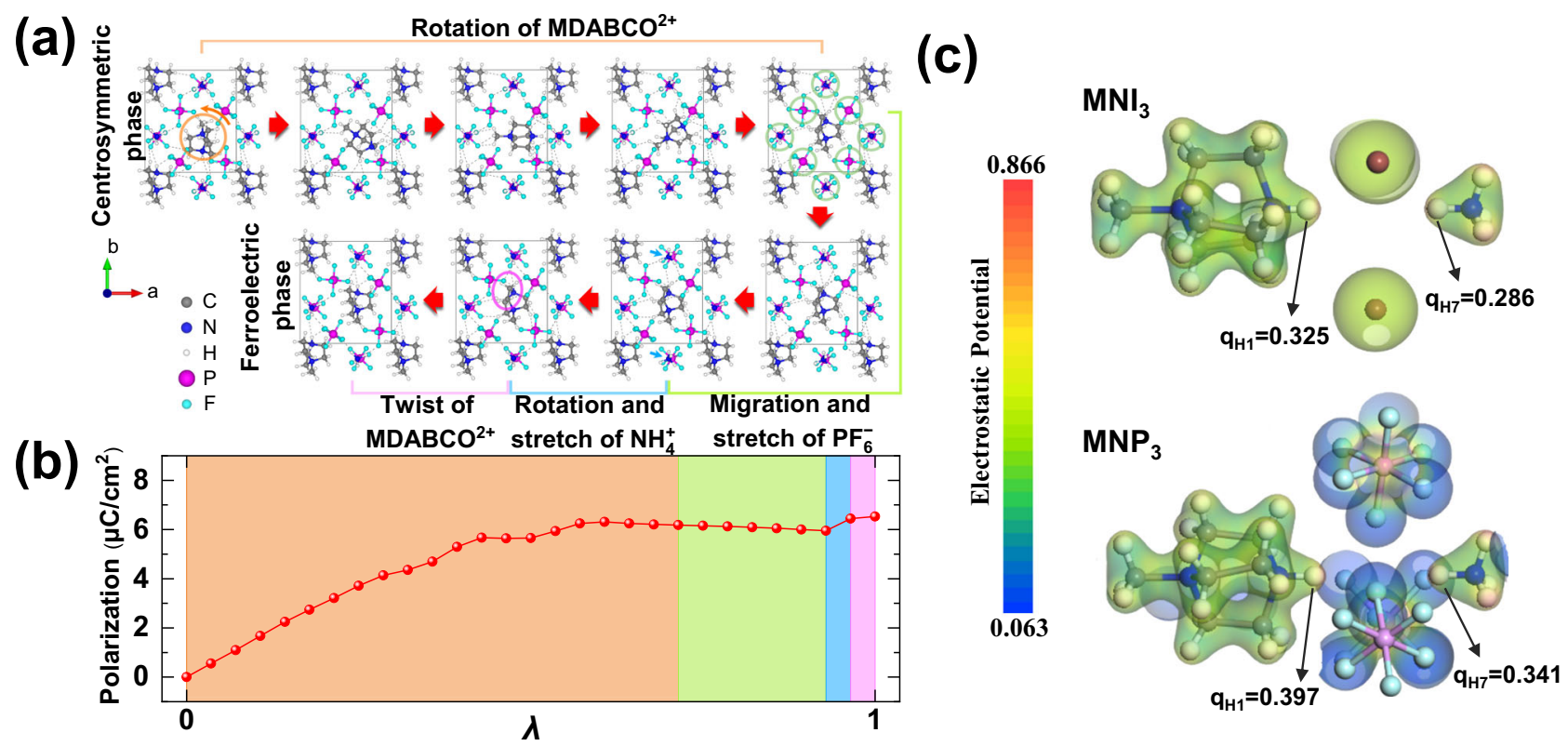

Fig. 5 Berry phase calculation of spontaneous polarization in $\mathbf{M N P}_{\mathbf{3}}$. a The dynamic path of ferroelectric phase transition in $M N P_{3}$. $\mathbf{b}$ The polarization value of $\mathrm{MNP}_{3}$ was obtained by Berry phase calculations. The path connects the centrosymmetric reference phase $(\lambda=0)$ to the ferroelectric phase $(\lambda=1)$. c Electrostatic potential of $\mathrm{MNI}_{3}$ and $\mathrm{MNP}_{3}$, and the Mulliken charges of $\mathrm{H}$ atoms participating in hydrogen bonds.

$110 \mathrm{kV} / \mathrm{cm}$. This enables a threshold voltage of $1 \mathrm{~V}$ for a $90 \mathrm{~nm}$ thick film, which complies with the miniaturization requirement of FeRAM devices. Both experimental results and DFT calculations provide robust evidence for the mechanism of MDABCO rotation upon ferroelectric switching and phase transition, the dynamics of which are modulated by the intermolecular hydrogen bonding interactions between $\mathrm{MDABCO}^{2+}$ and $\mathrm{PF}_{6}{ }^{-}$. This work demonstrates that regulating intermolecular interactions between the cation and anion can be used for the engineering of the coercive field in organic ferroelectric materials.

\section{Methods}

Synthesis. Detailed synthetic methods for preparing precursors are provided in Supplementary Information. For growing $\mathrm{MNP}_{3}$ crystal, $209 \mathrm{mg}(0.5 \mathrm{mmol})$ of MDABCO- $\left(\mathrm{PF}_{6}\right)_{2}$ and $81.5 \mathrm{mg}(0.5 \mathrm{mmol})$ of $\mathrm{NH}_{4} \mathrm{PF}_{6}$ were dissolved in acetonitrile until saturated, and the parallelogrammic single-crystals were grown after slow evaporation.

Characterization. All chemicals were purchased from Sigma-Aldrich without further purification. ${ }^{1} \mathrm{H}$ and ${ }^{13} \mathrm{C}$ nuclear magnetic resonance (NMR) was taken by AVII $400 \mathrm{MHz}$ NMR spectrometer of Bruker. Thermogravimetric analyses (TGA) were performed under a nitrogen atmosphere with a heating rate of $10^{\circ} \mathrm{C} / \mathrm{min}$ using a TA Instruments Trios V3.1 thermogravimetric analyzer. Differential scanning calorimetry (DSC) scans were performed under a nitrogen atmosphere with a heating rate of $10^{\circ} \mathrm{C} / \mathrm{min}$ using Mettler-Toledo DSC. The dielectric constant was measured by CVU unit in Keithley-SCS4200 with the pelleted sample. Powder X-ray diffraction (PXRD) patterns were recorded on a Bruker D8 Focus Powder $\mathrm{X}$-ray diffractometer using $\mathrm{Cu} \mathrm{K} \alpha$ radiation $(40 \mathrm{kV}, 40 \mathrm{~mA})$ at room temperature. Ferroelectric $P$ - $E$ curve was measured with Precision Multiferroic II Ferroelectric Test System of the Radiant Technologies with high voltage amplifier. Piezoresponse force microscopy (PFM) tests were performed on Bruker Dimension Icon Atomic Force Microscope with grown crystals or spray-coated samples on ITO.

Density functional theory (DFT) calculations. We performed the Berry phase calculations ${ }^{41,42}$ within the DFT framework as implemented in the Vienna ab initio simulation package (VASP) ${ }^{43,44}$. The exchange-correlation interactions were treated within the Perdew-Burke-Ernzerh (PBE) generalized gradient approximation ${ }^{45}$. To complement the deficiencies of DFT in treating dispersion interactions, the third-generation (D3) van der Waals corrections proposed by Grimme $^{46}$ were employed. The plane-wave cutoff energy was set to $520 \mathrm{eV}$, and the $k$-point mesh to $3 \times 3 \times 4$. The polarization was calculated using a supercell twice the size of the unit cell so that a centrosymmetric reference phase can be constructed. A convergence threshold of $0.01 \mathrm{eV} / \AA$ in force was reached in structural optimization. Electrostatic potential and Mulliken charge were calculated by $\mathrm{DMol} 3^{47,48}$ code in Materials Studio using a double numerical polarized basis set and $\mathrm{PBE}^{45}$ exchange-correlation functional.

Reporting summary. Further information on research design is available in the Nature Research Reporting Summary linked to this article.

\section{Data availability}

All data generated and analyzed in this study are included in the Article and its Supplementary Information, and are also available from corresponding authors upon request. Crystallographic data for this paper can be obtained free of charge from the Cambridge Crystallographic Data Centre via www.ccdc.cam.ac.uk/data_request/cif. CCDC- $2085249\left(\mathrm{MNP}_{3}\right.$ at RT) and CCDC- 2085250 (D-MNP ${ }_{3}$ at RT).

Received: 4 November 2021; Accepted: 19 January 2022; Published online: 10 February 2022

\section{References}

1. Whatmore, R. W., You, Y.-M., Xiong, R.-G. \& Eom, C.-B. 100 years of ferroelectricity-a celebration. APL Mater. 9, 070401 (2021).

2. Peña, M. A. \& Fierro, J. L. G. Chemical structures and performance of perovskite oxides. Chem. Rev. 101, 1981-2018 (2001).

3. Mischenko, A. S., Zhang, Q., Scott, J. F., Whatmore, R. W. \& Mathur, N. D. Giant electrocaloric effect in thin-film PbZr0.95Ti0.05O3. Science 311, 1270-1271 (2006).

4. Horiuchi, S. \& Tokura, Y. Organic ferroelectrics. Nat. Mater. 7, 357-366 (2008).

5. Li, W. et al. Chemically diverse and multifunctional hybrid organic-inorganic perovskites. Nat. Rev. Mater. 2, 16099 (2017).

6. Wei, L., Stroppa, A., Wang, Z. M. \& Gao, S. In Hybrid Organic-Inorganic Perovskites. (Wiley-VCH, 2020).

7. Song, X., Hodes, G., Zhao, K. \& Liu, S. Metal-free organic halide perovskite: a new class for next optoelectronic generation devices. Adv. Energy Mater. 11, 2003331 (2021).

8. $\mathrm{Li}, \mathrm{K}$. et al. Electronic structures and elastic properties of a family of metal-free perovskites. Mater. Chem. Front. 3, 1678-1685 (2019).

9. Bremner, C. A., Simpson, M. \& Harrison, W. T. A. New molecular perovskites: cubic $\mathrm{C} 4 \mathrm{~N} 2 \mathrm{H} 12 \cdot \mathrm{NH} 4 \mathrm{Cl} 3 \cdot \mathrm{H} 2 \mathrm{O}$ and $2-\mathrm{H}$ hexagonal C6N2H14.NH4Cl3. J. Am. Chem. Soc. 124, 10960 (2002).

10. Hang, T., Zhang, W., Ye, H.-Y. \& Xiong, R.-G. Metal-organic complex ferroelectrics. Chem. Soc. Rev. 40, 3577-3598 (2011). 
11. Shi, P.-P. et al. Symmetry breaking in molecular ferroelectrics. Chem. Soc. Rev. 45, 3811-3827 (2016).

12. Zhang, W. \& Xiong, R.-G. Ferroelectric metal-organic frameworks. Chem. Rev. 112, 1163-1195 (2012).

13. Zhang, H.-Y., Tang, Y.-Y., Shi, P.-P. \& Xiong, R.-G. Toward the targeted design of molecular ferroelectrics: modifying molecular symmetries and homochirality. Acc. Chem. Res. 52, 1928-1938 (2019).

14. Ye, H.-Y. et al. Metal-free three-dimensional perovskite ferroelectrics. Science 361, 151 (2018).

15. Wang, J.-J., Fortino, D., Wang, B., Zhao, X. \& Chen, L.-Q. Extraordinarily large electrocaloric strength of metal-free perovskites. Adv. Mater. 32, 1906224 (2020).

16. Mikolajick, T. et al. Next generation ferroelectric materials for semiconductor process integration and their applications. J. Appl. Phys. 129, 100901 (2021).

17. Park, M. H. et al. Ferroelectricity and antiferroelectricity of doped thin HfO2based films. Adv. Mater. 27, 1811-1831 (2015).

18. Fan, Z., Chen, J. \& Wang, J. Ferroelectric HfO2-based materials for nextgeneration ferroelectric memories. J. Adv. Dielectr. 06, 1630003 (2016).

19. Samara, G. A. The effects of deuteration on the static ferroelectric properties of KH2PO4 (KDP) †. Ferroelectrics 5, 25-37 (1973).

20. Nayeem, J., Wakabayashi, H., Kikuta, T., Yamazaki, T. \& Nakatani, N. Ferroelectric properties of deuterated glycine phosphite. Ferroelectrics 269, 153-158 (2002).

21. Horiuchi, S., Kumai, R. \& Tokura, Y. A supramolecular ferroelectric realized by collective proton transfer. Angew. Chem. Int. Ed. 46, 3497-3501 (2007).

22. Ubbelohde, A. R. \& Gallagher, K. J. Acid-base effects in hydrogen bonds in crystals. Acta Crystallogr. 8, 71-83 (1955).

23. Zhang, W.-Y. et al. Precise molecular design of high-Tc 3D organic-inorganic perovskite ferroelectric: [MeHdabco]RbI3 (MeHdabco = N-Methyl-1,4diazoniabicyclo[2.2.2] octane). J. Am. Chem. Soc. 139, 10897-10902 (2017).

24. Loiacono, G. M., Balascio, J. F. \& Osborne, W. Effect of deuteration on the ferroelectric transition temperature and the distribution coefficient of deuterium in $\mathrm{K}(\mathrm{H} 1-\mathrm{xDx}) 2 \mathrm{PO} 4$. Appl. Phys. Lett. 24, 455-456 (1974).

25. Shi, C., Zhang, X., Yu, C.-H., Yao, Y.-F. \& Zhang, W. Geometric isotope effect of deuteration in a hydrogen-bonded host-guest crystal. Nat. Commun. 9, 481 (2018).

26. Horiuchi, S., Ishibashi, S., Kobayashi, K. \& Kumai, R. Coexistence of normal and inverse deuterium isotope effects in a phase-transition sequence of organic ferroelectrics. RSC Adv. 9, 39662-39673 (2019).

27. Ai, Y., Lv, H.-P., Wang, Z.-X., Liao, W.-Q. \& Xiong, R.-G. H/F substitution for advanced molecular ferroelectrics. Trends Chem. 3, 1088-1099 (2021).

28. Whatmore, R. In Springer Handbook of Electronic and Photonic Materials. (Springer International Publishing, 2017).

29. Harris, R. K. \& Olivieri, A. C. Quadrupolar effects transferred to spin-12 magic-angle spinning spectra of solids. Prog. Nucl. Magn. Reson. Spectrosc. 24, 435-456 (1992).

30. Gobetto, R. et al. 1H MAS, 15N CPMAS, and DFT investigation of hydrogenbonded supramolecular adducts between the diamine 1,4-diazabicyclo-[2.2.2] octane and dicarboxylic acids of variable chain length. Chem. Mater. 17, 1457-1466 (2005).

31. Harada, J. et al. Directionally tunable and mechanically deformable ferroelectric crystals from rotating polar globular ionic molecules. Nat. Chem. 8, 946-952 (2016).

32. Harada, J. et al. Ferroelectricity and piezoelectricity in free-standing polycrystalline films of plastic crystals. J. Am. Chem. Soc. 140, 346-354 (2018).

33. Harada, J. et al. Plastic/ferroelectric crystals with easily switchable polarization: low-voltage operation, unprecedentedly high pyroelectric performance, and large piezoelectric effect in polycrystalline forms. J. Am Chem. Soc. 141, 9349-9357 (2019).

34. Harada, J. Plastic/ferroelectric molecular crystals: ferroelectric performance in bulk polycrystalline forms. APL Mater. 9, 020901 (2021).

35. Das, S., Mondal, A. \& Reddy, C. M. Harnessing molecular rotations in plastic crystals: a holistic view for crystal engineering of adaptive soft materials. Chem. Soc. Rev. 49, 8878-8896 (2020).

36. Stephan, K. \& Peter, L. Ferroelectric polarization in multiferroics. Phys. Sci. Rev. 4, 20190015 (2019).

37. Song, X.-J. et al. Bistable state of protons for low-voltage memories. J. Am. Chem. Soc. 142, 9000-9006 (2020).

38. Foster, C. M. et al. Single-crystal $\mathrm{Pb}(\mathrm{ZrxTi1}-\mathrm{x}) \mathrm{O} 3$ thin films prepared by metal-organic chemical vapor deposition: Systematic compositional variation of electronic and optical properties. J. Appl. Phys. 81, 2349-2357 (1997).

39. Chen, X., Han, X. \& Shen, Q.-D. PVDF-based ferroelectric polymers in modern flexible electronics. Adv. Electron. Mater. 3, 1600460 (2017).

40. Aizu, K. Possible species of "Ferroelastic" crystals and of simultaneously ferroelectric and ferroelastic crystals. J. Phys. Soc. Jpn. 27, 387-396 (1969).
41. Vanderbilt, D. \& King-Smith, R. D. Electric polarization as a bulk quantity and its relation to surface charge. Phys. Rev. B 48, 4442-4455 (1993).

42. King-Smith, R. D. \& Vanderbilt, D. Theory of polarization of crystalline solids. Phys. Rev. B 47, 1651-1654 (1993).

43. Kresse, G. \& Furthmüller, J. Efficient iterative schemes for ab initio totalenergy calculations using a plane-wave basis set. Phys. Rev. B 54, 11169-11186 (1996).

44. Kresse, G. \& Furthmüller, J. Efficiency of ab-initio total energy calculations for metals and semiconductors using a plane-wave basis set. Comput. Mater. Sci. 6, 15-50 (1996).

45. Perdew, J. P., Burke, K. \& Ernzerhof, M. Generalized gradient approximation made simple. Phys. Rev. Lett. 77, 3865-3868 (1996).

46. Grimme, S., Antony, J., Ehrlich, S. \& Krieg, H. A consistent and accurate ab initio parametrization of density functional dispersion correction (DFT-D) for the 94 elements H-Pu. J. Chem. Phys. 132, 154104 (2010).

47. Delley, B. An all-electron numerical method for solving the local density functional for polyatomic molecules. J. Chem. Phys. 92, 508-517 (1990).

48. Delley, B. From molecules to solids with the DMol3 approach. J. Chem. Phys. 113, 7756-7764 (2000).

\section{Acknowledgements}

K.P.L. would like to acknowledge the Singapore's Ministry of Education Tier 2 grant (MOE2019-T2-1-037), the Shenzhen Peacock Plan (Grant No. KQTD2016053112042971). F.P. would like to acknowledge the Guangdong Special Support Program, and Soft Science Research Project of Guangdong Province (No. 2017B030301013). K.Y. would like to acknowledge partial supports by A*STAR, Singapore, RIE2020 Advanced Manufacturing and Engineering (AME) Programmatic Fund, (Grant No. A20G9b0135).

\section{Author contributions}

H.S.C. designed, synthesized and characterized the $\mathrm{MNP}_{3}$ crystals. K.P.L. supervised the research. S.L., S.Z. and F.P. did DFT calculations. I.H.P. did a single-crystal XRD structure analysis. W.H.L. and K.Y. did a ferroelectric experiment and analysis. Z.Z. and Q.H.X. did a temperature-dependent SHG experiment. K.C.K. did PFM measurement. L.W. did temperature-dependent dielectric measurement. I.H.O. did an analysis on the deuteration effect. H.S.C., S.L. and K.P.L. wrote the manuscript in consultation with all authors. H.S.C. and S.L. contributed equally.

\section{Competing interests}

The authors declare no competing interests.

\section{Additional information}

Supplementary information The online version contains supplementary material available at https://doi.org/10.1038/s41467-022-28314-8.

Correspondence and requests for materials should be addressed to Feng Pan or Kian Ping Loh.

Peer review information Nature Communications thanks the other anonymous reviewer(s) for their contribution to the peer review of this work. Peer reviewer reports are available.

Reprints and permission information is available at http://www.nature.com/reprints

Publisher's note Springer Nature remains neutral with regard to jurisdictional claims in published maps and institutional affiliations.

Open Access This article is licensed under a Creative Commons Attribution 4.0 International License, which permits use, sharing, adaptation, distribution and reproduction in any medium or format, as long as you give appropriate credit to the original author(s) and the source, provide a link to the Creative Commons license, and indicate if changes were made. The images or other third party material in this article are included in the article's Creative Commons license, unless indicated otherwise in a credit line to the material. If material is not included in the article's Creative Commons license and your intended use is not permitted by statutory regulation or exceeds the permitted use, you will need to obtain permission directly from the copyright holder. To view a copy of this license, visit http://creativecommons.org/ licenses/by/4.0/

(C) The Author(s) 2022 